\section{Neutropenieprophylaxe noch nicht ausgereizt}

In einer aktuellen Studie zur Prophylaxe chemotherapieinduzierter und febriler Neutropenien wurde neben den in den Leitlinien beschriebenen Parametern auch eine Neutropenie im vorhergehenden Zyklus berücksichtigt.

$\mathrm{D}$ ie evidenzbasierten Leitlinien von EORTC/NCCN empfehlen die Prophylaxe einer chemotherapieinduzierten Neutropenie (CIN) und febrilen Neutropenie (FN) abhängig von der relativen Myelotoxizität des Chemotherapieregimes und dem Vorliegen von Risikofaktoren (Indikation zur G-CSF-Prophylaxe bei Patienten ohne Risikofaktoren ab einem FN-Risiko $\geq 20 \%$, bei Patienten mit Risikofaktoren bei einem FN-Risiko von 10-20\%). In der prospektiven Beobachtungsstudie MONITOR-GCSF wurde die Prophylaxe mit dem Filgrastim-Biosimilar Ep2006 bei 1.447 Patienten untersucht, die bis zu 6 Zyklen einer myelosuppressi- ven Chemotherapie erhielten. Die EORTC-Leitlinien wurden so angepasst, dass Patienten mit niedrigem $(<10 \%)$ oder mittlerem (10-20\%) CIN-/FN-Risiko eine Sekundärprophylaxe erhielten, wenn sie eine CIN- oder FN-Episode im vorhergehenden Zyklus durchgemacht hatten. Zudem war keine Prophylaxe vorgesehen für Patienten, die eine Chemotherapie mit hoher $(\geq 20 \%)$ oder mittlerer (10-20\%) Myelotoxizität bei gleichzeitigem Vorliegen von Risikofaktoren erhielten sowie nicht für Patienten mit niedriger oder mittlerer Myelotoxizität, die bisher keine CIN/FN hatten. Dementsprechend bekamen 56,6\% der Patienten die korrekte CIN-/FN-Prophylaxe, 17,4\% waren unter-, $26,0 \%$ übertherapiert.

Bei Patienten mit Überprophylaxe waren CIN (alle Grade), FN und Hospitalisierungen aufgrund von CIN seltener als bei Patienten mit Unter- oder leitliniengerechter Prophylaxe. Patienten mit Unterund leitliniengerechter Prophylaxe unterschieden sich nur hinsichtlich CIN/FNassoziierter Chemotherapiebeeinträchtigungen (bei Untertherapierten häufiger).

Fazit: Diese Daten aus dem Alltag zeigen bessere Ergebnisse hinsichtlich CIN, FN und Hospitalisierungen, wenn die Patienten über den Leitlinienempfehlungen behandelt werden. Judith Neumaier

Bokemeyer $\mathrm{C}$ et al. Over- and under-prophylaxis for chemotherapy-induced (febrile) neutropenia relative to evidence-based guidelines is associated with differences in outcomes: findings from the MONITOR-GCSF study. Support Care Cancer. 2017;25(6):1819-28.

\title{
Tool zur CINV-Vorhersage
}

Trotz effektiver Antiemetika und evidenzbasierter Leitlinien leiden bis zu $40 \%$ der Krebspatienten unter Chemotherapie nach wie vor unter Übelkeit und Erbrechen. Ein neues Vorhersagemodell hilft, das Risiko besser abzuschätzen.

B isherige Modelle zur Vorhersage des Risikos für chemotherapieinduzierte Übelkeit und Erbrechen (CINV) haben Limitationen, daher wurden ihre Daten nun kombiniert ausgewertet, um ein Vorhersagemodell $\mathrm{zu}$ entwickeln, mithilfe dessen das individuelle Risiko für CINV vom Grad $\geq 2$ an den ersten 5 Ta- gen einer Chemotherapie in jedem Zyklus berechnet werden soll. Ausgewertet wurden die Daten von 1.198 Patienten aus 5 nicht interventionellen Studien zur CINV-Prophylaxe, die insgesamt 4.197 Chemotherapiezyklen erhalten hatten.

$22,8 \%$ der Patienten litten an Erbrechen, $46,9 \%$ an Übelkeit in den ersten 24

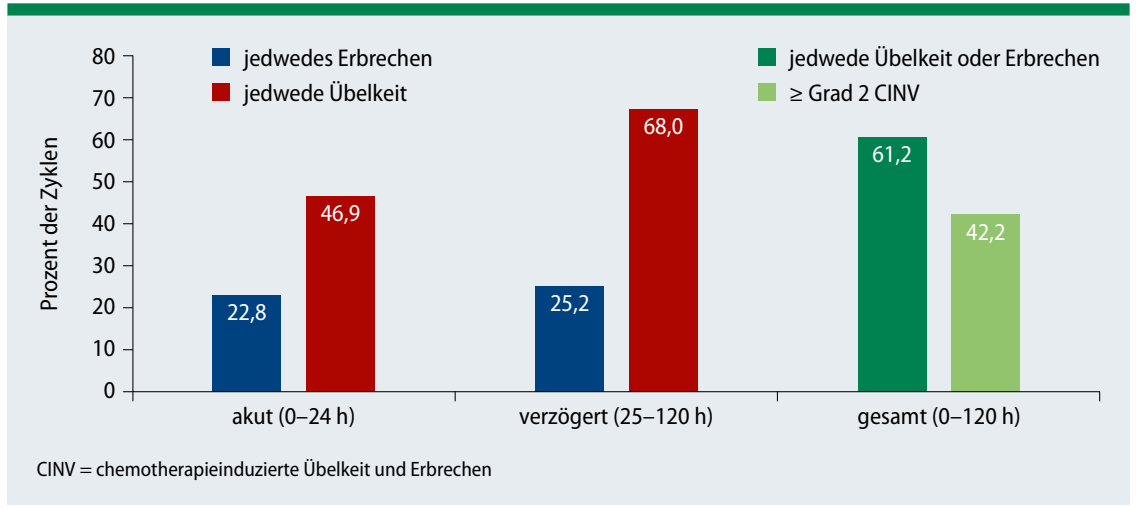

Abb. 1: Übelkeit und Erbrechen im Rahmen der 4.197 analysierten Zyklen.
Stunden nach Beginn der Chemotherapie. Der Anteil an Patienten mit verzögerten Symptomen (Tage 2-5) betrug 25,2\% (Erbrechen) und 68,0\% (Übelkeit). 42,2\% der Patienten hatten CINV vom Grad $\geq 2$ (Abb. 1).

8 Risikofaktoren wurden identifiziert: Alter $<60$ Jahre, 1. und 2. Chemotherapiezyklus, erwartete Übelkeit und Erbrechen, morgendliche Übelkeit in der Vorgeschichte, weniger als 7 Stunden Schlaf in der vorhergehenden Nacht, CINV im vorhergehenden Zyklus, Selbstmedikation und die Anwendung von platin- oder anthrazyklinbasierten Regimes. Aus diesen 8 Risikofaktoren wurde ein Risikoscore von 0-32 entwickelt, wobei ein höherer Score ein höheres Risiko für CINV in den ersten $5 \mathrm{Ta}$ gen der Chemotherapie bedeutet. Die Analyse zeigte eine gute prädiktive $\mathrm{Ge}$ nauigkeit des Scores (AUC 0,69).

Fazit: Dieser Risikoscore ist einfach anzuwenden und ermöglicht es, Patienten mit hohem CINV-Risiko zu identifizieren.

Judith Neumaier

Dranitsaris G et al. The development of a prediction tool to identify cancer patients at high risk for chemotherapyinduced nausea and vomiting. Ann Oncol. 2017;28(6):1260-7. 